\title{
ORIENTACIÓN PROFESIONAL DEL DOCENTE A PARTIR DE LA INVESTIGACIÓN ACCIÓN. CASO: CONSTRUCCIÓN DE UN PROYECTO DE FORMACIÓN EN VALORES
}

\author{
Esther Fragoso Fernández \\ Docente investigadora \\ Universidad La Salle \\ Pachuca, México
}

Recibido 8-XI-2007 • Aceptado 13-XI-2007 • Corregido 07-III-2008

\begin{abstract}
Resumen: El trabajo que se presenta es un avance de dos de las tres fases que conforman la presente investigación. En este estudio se aprecian los lineamientos teóricos que sustentan el proyecto de investigación y se valora la utilización de la investigación acción como metodología para la formación continua del profesorado, muestra el desarrollo que se llevó a cabo en el colegio, así como, las ventajas y obstáculos que se presentaron a lo largo de su puesta en práctica. En la metodología se contempla la aplicación de la investigación acción como una herramienta de formación del profesorado del Colegio Montessori Tepuente en Cuernavaca Morelos, México, al desarrollar una propuesta educativa en valores para la comunidad escolar. Los resultados reflejan el avance de los instrumentos trabajados con maestros, padres de familia, alumnos y directivos del plantel. La aportación central de esta investigación muestra una versión a nivel descriptivo de la orientación y desarrollo logrado con los maestros a partir de la investigación acción participativa en la elaboración de un programa en valores para todos los ámbitos del colegio.

En el desarrollo de la investigación se está aplicando la tercera fase de dicho programa como prueba piloto en el ciclo escolar 2007-2008. Con la investigación se espera dar soporte formativo y académico a la comunidad educativa (directivos, maestros, padres y niños del colegio en particular) y a futuro, considerar este trabajo como un modelo para que otras escuelas afines se beneficien con el programa de valores en el colegio.
\end{abstract}

Palabras clave: Investigación acción, docente, valores.

\section{Introducción}

La socialización se da en el individuo en dos fases: primaria y secundaria. La socialización primaria se efectúa durante la niñez y se desarrolla habitualmente en el seno de la familia, una de sus características es la carga afectiva con la que se transmiten los contenidos y la identificación con el mundo tal y como lo presentan los adultos. "El niño internaliza el mundo de acuerdo a los parámetros que le ofrece la familia como el único existente y que puede concebir" (Loera, 1999, p. 33 en Martínez y Hernández, 2005), de aquí la necesidad de que los padres cuenten con información suficiente para proveer a sus hijos de este primer acercamiento al mundo real.

La socialización primaria -desarrollada principalmente en familia- no es universal ni permanece estática; la segunda fase de la socialización que reciben los niños ocurre en la escuela. En ella, se inserta el individuo ya socializado a nuevos sectores del mundo objetivo de su sociedad. Sin embargo, los resultados insuficientes 


\begin{abstract}
This presentation is a preliminary report of a larger research project which focuses on the application of action research as a tool for teacher training at the Colegio Montessori Tepuente in Cuernavaca, Morelos; the goal is to develop an education-based proposal on values for the school community.

This study reviews the theoretical underpinnings which support the project and evaluate the use of action research as a method for continuous teacher training and its development as undertaken in the school as well as the advantages and difficulties which arose throughout the implementation at the school. The central contribution of this investigation shows a descriptive level version of the orientation and development achieved with the teachers through the program in values which was designed for all aspects of the school.
\end{abstract}

Key words: Action investigation, teachers, values. que se perciben a través de la conducta de los niños de la actual sociedad muestran lo que Loera comenta: "los programas de valores familiarizan a los alumnos con los nombres de los valores pero no modifican la estructura social y las conductas" (Loera, 1999, p. 33 en Martínez y Hernández, 2005). Ampliando el proceso de socialización al entorno social de la familia y escuela donde el niño convive, Guy Rocher lo extiende al "proceso por cuyo medio la persona humana aprende e interioriza, en el transcurso de su vida, los elementos socio-culturales de su medio ambiente, los integra a la estructura de su personalidad ... y se adapta así al entorno social en cuyo seno debe vivir" (Rocher, 1983, p. 133).

El concepto fundamental de la educación en valores que aquí se enmarca, proviene de la etimología fonética y morfológica educare que significa conducir, guiar, orientar y la etimología semántica educere que significa hacer salir, extraer, dar a luz (cfr. Aula Santillana, 1996, p. 475) ambos términos integran el significado de intervenir intencionalmente en el desarrollo del ser humano con el fin de que alcance su perfeccionamiento en la óptima realización de sus potencialidades. Tarea fundamental de la familia y la escuela, que en tiempos de grandes cambios educativos, familiares y sociales, no fácil afrontar.

La enseñanza escolarizada como ejercicio reflexivo de la propia práctica para conocerla y, a partir de ello, crear estrategias para mejorarla, redundaría en una especialización del docente en su labor, logrando con ello mayor calidad educativa en los procesos y resultados con sus alumnos.

Es así, que el objetivo de esta investigación tomó como punto de partida la situación educativa y de formación de los alumnos de una escuela primaria y el nivel de desarrollo y comprensión de los profesores de esta institución sobre su propia práctica educativa.

El trabajo de investigación se llevó a cabo en el Colegio Montessori de Tepuente, una escuela de educación básica privada, 
cuya población infantil es de 78 alumnos y la población docente se conforma de 7 guías y asistentes, 1 directivo y 4 profesores de asignaturas extracurriculares. Este colegio se encuentra en la ciudad de Cuernavaca, Morelos, México y posee una antigüedad de 8 años.

\section{Referencia a estudios afines al que aquí se presenta}

En este apartado se presentan algunos trabajos de investigación realizados en torno a la temática de la educación en valores en diferentes ámbitos educativos, haciendo con ellos referencia a investigaciones afines a la presente.

En un estudio llevado a cabo por Cárdenas y Delgado (en Hirsch, 2006) sobre la educación valoral en la escuela primaria a inicios del siglo XXI bajo el enfoque cognitivo-evolutivo de Kohlberg y Piaget muestran que "los valores son orientaciones generales de la conducta que se encuentran encarnados en sistemas de preferencia, patrones de interacción y sistemas de regulación del comportamiento social, y que a su vez son constitutivos de una comunidad. Las orientaciones valorativas se aprenden en el transcurrir de la vida cotidiana y forma parte fundamental del sentido de la acción humana. Por lo que sugieren que en todo estudio valoral es necesario prestar atención a las prácticas sociales y al transcurrir de la vida cotidiana, ya que son las claves para comprender la acción individual y social. Lo anterior determinó que en la investigación que se presenta buscara un acercamiento con la realidad cotidiana que viven los alumnos de la escuela primaria y su relación con el proceso de formación valoral.

Por otro lado, Cárdenas menciona que dentro de las pautas que regularon la acción docente se identificó en la parte central del proceso de investigación el conocimiento del discurso del profesor sobre su propia práctica educativa. En relación con este trabajo se realizaron dos tipos de actividades: primero se hicieron observaciones durante la clase para identificar cuáles eran las recomendaciones más frecuentes que surgían y la justificación que se daba a las reglas de conducta y a las múltiples decisiones que debían tomarse en el desarrollo de las actividades. Una de las conclusiones que se toma en cuenta de este trabajo realizado, es que la observación de la vida cotidiana en las aulas permite fortalecer la tesis de que más allá del saber declarativo, los valores se transmiten gradual y constantemente mediante una gran cantidad de eventos cuya lógica singular tiende a complementarse.

También se revisó una investigación del programa de Valores Humanos Sathya Sai que se encuentra en su fase II donde se ofrece información sobre el avance del plan piloto del programa de educación en valores humanos 2006-2007 adscrito al Centro de Investigación en Educación Infantil (CIEDIN) y a la Cátedra de Educación para la Paz de la Universidad Pedagógica Experimental Libertador en la UNESCO (2006). Aquí se reporta que se logró - a partir del registro de situaciones observadas- la elección de dos escuelas en las que se estudian las interacciones familiares y la violencia intrafamiliar con relación al bajo rendimiento académico de los niños. En este momento el estudio se encuentra en la fase de diseño de un programa de entrenamiento y acompañamiento para brindar herramientas a las madres de familia para contemplar su participación efectiva en el proceso de enseñanza aprendizaje de sus hijos; todo ello como respuesta al vínculo necesario familia-escuela para afrontar juntos el proceso de desarrollo integral de los alumnos y unir múltiples acciones que redunden en el mejoramiento de su socialización y del incremento del nivel académico. Esta es una de las investigaciones encontradas sobre este programa de valores que en la actualidad se desarrolla en otra escuela Sai en Latinoamérica. 
En la revisión de Díaz (2006) se puede apreciar el análisis del problema de la formación de valores en relación al currículo. En este trabajo se propone que el análisis se ha de realizar desde tres ángulos: el primero considerando la perspectiva del currículo oculto, el segundo a partir de la propuesta constructivista y el tercero considerando la estrategia de elaboración de los temas transversales. Para este trabajo resulta importante el énfasis en el primer rubro, porque guarda una estrecha relación entre la esfera de lo valoral y la actitudinal. El currículo oculto tiene que ver más con lo que se hace que con lo que se dice. En muchas ocasiones los códigos empleados en la comunicación -forma de decir algo- no converge con lo que se expresa en el plano explícito; es así que afirma Díaz (2006, p. 4) "las actuaciones de diversos actores de la comunidad escolar responden a concepciones nucleares que tiene un sujeto y son percibidas por los estudiantes en un plano distinto al conciente y que es mucho más eficaz".

En la revisión de un texto de Latapí (2004) se presenta un análisis sobre desarrollo histórico de los diferentes programas de formación en valores impulsados por los gobiernos mexicanos desde la educación moral del siglo XIX hasta la gestión del presidente Vicente Fox (20002006), donde se muestra puntualmente las diferentes posturas de los programas, los conflictos que han surgido en su aplicación y se visualiza a futuro la manera de integrar la formación en valores en las políticas públicas. Este ha sido un texto fundamental para la investigación que aquí se propone.

Otro programa de formación en valores dirigido a padres de familia que está haciendo resonancia en México es que se conoce como "Padres Eficaces con Entrenamiento Sistemático" (PECES) diseñado en España. Un equipo de investigación dirigido por Dinkmeyer y Muckay (1988, en Bartau et al., s.f.) presenta diversos métodos para mejorar la relación familiar, en donde se resalta la capacidad de intervención de la escuela en la formación de los padres; muestran resultados muy alentadores en la vida de los niños cuando el colegio y la familia trabajan en una misma dirección.

Elexpuro y Medrano (2001) presentan una investigación denominada: "Desarrollo de los valores en las instituciones educativas" de la Universidad de Deusto, cuyo objetivo es realizar un acercamiento a los valores del profesorado, los alumnos y de los centros, de tal forma que se integren los valores desde una perspectiva global. En esta investigación los instrumentos utilizados fueron escalas de observación y cuestionarios para evaluar cada valor. Las aportaciones más importantes de sus resultados consistieron en identificar los valores de cada grupo de estudio, a partir de los cuales se diseñó una propuesta global institucional que contiene una visión compartida del trabajo con los valores. Sin duda este trabajo ofrece un sustento teórico metodológico importante en diferentes momentos de nuestra evaluación y construcción de la propuesta.

En la investigación realizada por Pouljol (en Yurén, Navia y Saenger, 2005) se concluye que en las estrategias de formación docente para la eticidad, hay que tomar en cuenta la formación para la relación y que resulta prioritario que los sujetos se interroguen a sí mismos sobre el marco de la intersubjetividad.

Martínez y Galíndez (2003, p. 9) en colaboración con la Universidad de Deusto proponen un "Proyecto de Calidad Integrado" que parte de las conclusiones obtenidas por los investigadores. Algunas que se consideran relevantes consideran tres elementos fundamentales para el presente estudio: $1^{\circ}$. Concluyen que la implicación de los padres en las escuelas afecta positivamente al rendimiento académico de los hijos; $2^{\circ}$. Reportan que los beneficios que se obtienen no sólo benefician a los padres y los estudiantes, encontraron que 
también repercuten en los profesores y en el propio centro escolar; $3^{\circ}$. Encontraron que existen diferentes aproximaciones para comprender la involucración de los padres a nivel escolar. La investigación apuesta hacia una aproximación centrada en la participación.

Ortega y Míngues (2001) presenta una propuesta de integración de los valores en el currículo, en él afirman que no depende tanto de la vinculación de los valores a unos contenidos, sino a las estructuras relacionales en el aula, al estilo de enseñanza y del clima moral de la clase. Los aprendizajes de los valores no acompañan automáticamente a los contenidos curriculares concretos; sino que lo más decisivo para dicho aprendizaje es el espíritu que imprime el profesor a su tarea docente. El modo, estilo y ethos del profesor facilita el aprendizaje moral de los alumnos. Estas conclusiones resultan valiosas como ejes importantes que se han tomado en cuenta para la investigación que aquí se expone.

Se hizo la revisión de dos textos sobre valores publicados por la Secretaría de Educación Pública de México (SEP) a nivel primaria: el primero, 200 dias de reflexiones se ofrece como un material de lectura para reforzar la práctica docente; está constituido por cuentos y leyendas populares y al final invita a la reflexión del alumno ofreciéndole la posibilidad de valorar y al mismo tiempo "significar su propia vida" (CREFAL/SEP, 2000). Esta estrategia del cuento es una herramienta importante como instrumento de evaluación diagnóstica. El segundo texto forma parte de una colección que edita la CREFAL/SEP (2000) donde se ofrece información a los padres y madres o familiares que están a cargo de los niños para que los puedan ayudar a tener un redimiendo escolar satisfactorio; en este libro se hace hincapié en la importancia que caminen juntos para conseguir un objetivo común la escuela y la familia, ya que con ello se logra mejorar la formación de los niños.

\section{La investigación vista desde el docente}

La actividad de la docencia y de la investigación comúnmente se perciben como dos actividades desvinculadas entre sí. En los programas de formación o actualización docente, los profesores se forman normalmente en una profundización de la asignatura que imparten o en la adquisición de algunas de las estrategias pedagógicas para la enseñanza de la misma, pero muy raras veces se incluye en estos programas la formación de los docentes en la investigación en general y en forma más precisa, en la investigación de su propia práctica.

Es necesario que el docente no solo conozca con precisión los contenidos académicos de las asignaturas que imparte, sino que al mismo tiempo maneje la metodología pedagógica necesaria para la enseñanza y para el aprendizaje de la asignatura con el fin de contar con los elementos requeridos para su transmisión y con las técnicas que ha de mostrar a sus alumnos para facilitar la adquisición de la información brindada por él.

En el proceso de enseñanza el docente es el protagonista primordial, ya que de su actuar diario depende en gran medida la calidad con la que se educa a los estudiantes, en ello interviene su compromiso como persona ante su labor docente, la calidad de su propia formación, la motivación para desarrollar su práctica, el nivel de identificación que desarrolle con la institución a la que pertenece, el tiempo con el que cuenta para desarrollar su actividad y el contexto socioeconómico que le rodea, entre otros. Es así que, "el propósito de la investigación acción en comparación con gran parte de la investigación tradicional es resolver los problemas diarios inmediatos de los profesionales en ejercicio" (Reyna, 2007, p. 21).

La tarea de los docentes, en ocasiones, se limita a planear su curso, impartir sus clases y elaborar los exámenes para generar las calificaciones de los alumnos; 
es requerido para el óptimo desenvolvimiento de su clase, que se elaboren inducciones que preparan a los alumnos al tema por estudiar, se valore el logro de los fines perseguidos, se realicen las adaptaciones según el contexto de cada grupo en específico, se brinden las estrategias por diferentes canales de aprendizaje para que se logre la apropiación del objeto de estudio, crear en cada sesión formas de evaluar el aprendizaje de ese pequeño momento del programa, proseguir o retroalimentar de acuerdo a los resultados, polemizar si la forma en que se organizaron los contenidos en la planeación fue la óptima. Ello se logra a través de un diálogo continuo con el propio hacer de cada sesión; en donde pueden intervenir el docente mismo, otros docentes de la misma asignatura o de otra asignatura que atienden al mismo grupo y el mismo grupo de alumnos que lo está viviendo.

Se impone la necesidad de reflexionar sobre la docencia como un ejercicio profesional que tiene características y exigencias específicas, que está más allá de la simple transmisión del conocimiento, es necesario, redimensionar el papel de la docencia como actividad profesional que requiere conocimientos teórico, metodológicos y habilidades investigativas para incorporar al docente activamente al trabajo profesional y no como receptor pasivo del conocimiento ya elaborado por otros (cfr. Reyna, 2007, pp. 15-16).

En algunas situaciones la institución promueve la evaluación del docente por parte de él mismo, de sus jefes y de sus alumnos o el mismo compromiso del docente lo lleva a querer mejor su práctica, sin embargo, esto se hace en muchos de los casos, desde la imaginación o copiando lo que han hecho otras instancias, sin mayor valoración de cómo o en qué circunstancias fueron aplicados los instrumentos de valoración que han llevado a transformar algunas acciones en el aula, pero si esto lo reproduce el profesor desde las mismas carencias formativas en la didáctica de enseñanza y los nulos conocimientos sobre la investigación de la propia práctica, no alcanzarán los mismos resultados. Se necesita formar en el ámbito investigativo de la propia labor, es decir: cómo elaborar un diagnóstico real de las necesidades en el aula, elegir referencias para enfrentar la problemática, crear objetivos, justificaciones y métodos ad hoc al problema y realizar un proyecto de intervención en el aula para el cambio de aquellas conductas detectadas, entre otras acciones (Cfr. Sañudo, 1997).

Otra de las situaciones mas frecuentes en la realidad mexicana es que al profesor se le pague un salario por las clases impartidas frente al grupo y no se le asigne un tiempo para la profundización de su práctica docente, esto provoca que el profesor tenga más trabajo al dedicar tiempo de elaboración de su clase y dificulta que desee optar por dedicarse a la investigación. Lo óptimo es dar espacios para la investigación de la propia docencia, práctica poco extendida en nivel superior.

\section{Situación de la docencia en la formación en valores}

A partir de un revisión inicial que se realizó sobre los diferentes proyectos de valores en educación básica, se afirma que por la indiferencia que muchos maestros muestran hacia la formación en valores, las escuelas mexicanas de educación básica atraviesan por una situación de grave deterioro. Varios estudios de campo llevados a cabo en espacios urbanos y rurales, coinciden en describir un panorama preocupante sobre el funcionamiento escolar en lo que concierne a la formación de valores (García y Vanilla, 1992; Rockwell, 1995; Manteca, 1999; Molina, 1999 en Latapí, 2004).

El tema de la transmisión de valores ha sido tratado en forma amplia y desde muchos sistemas educativos en la relación maestro-alumno dentro del ámbito escolar, sin embargo señala Schmelkes y otros (1995, p. 198) es "novedoso y por lo mismo, 
precario el tema de educación de la familia en los diferentes enfoques de investigación de campo, a la vez que indican nuevamente un fenómeno de dispersión del área, manifiestan la riqueza potencial del área temática en ciernes que no se encuentra ligada en un solo enfoque de investigación". Es por lo tanto un tema con importantes posibilidades de aportación que requiere de un trato científico para una comprensión más precisa de lo que sucede en su interior.

Esta misma necesidad de crear programas de educación en valores a partir del vínculo familia-escuela se encuentra reflejada en la propuesta de la Organización para la Cooperación y el Desarrollo Económico (2002, p. 162) en los resultados de PISA 2000 cuando sugiere que “...este análisis indica que el éxito académico podría estar relacionado con sinergias positivas entre el hogar y la escuela y que la comunicación entre padres e hijos puede ser de beneficio para los jóvenes en términos educativos. Un objetivo importante para las políticas públicas podría consistir en el trabajo con los padres, particularmente con aquellos cuyos logros educativos sean más limitados, con el fin de facilitar su interacción con los hijos y las escuelas a las que asisten de manera que mejore el aprendizaje de los jóvenes. Aunque no es tarea fácil, es de gran importancia que el apoyo de los padres sea visto como un accesorio clave para la educación de los hijos".

Es fundamental la aportación de este trabajo al diseñar un programa de formación en valores que esté integrado al proyecto educativo escolar construido a partir de la aportación de los docentes, con el fin de atender la situación de carencia de medios para desarrollar los valores, tanto en el colegio como los que vive la familia de hoy y con ello coadyuvar al desarrollo de una sociedad más sana y armónica.

La dificultad no termina ahí, ya que existe una disociación entre lo que se enseña en la familia y lo que el centro escolar determina como valioso, esto representa para los niños una contradicción entre lo que se afirma que es bueno en casa y lo que los docentes transmiten como valor; provocando en ellos graves confusiones y una pérdida de autoridad de ambos educadores.

Existen otros agentes socializadores que en la actualidad cobran cada vez mayor importancia en la vida de los niños. Esto hace referencia a la transmisión de valores que producen los diferentes ámbitos sociales a los que los pequeños pertenecen, y que -de manera formal e informal, planificada o no- educan de acuerdo a sus valores:

a) Una formación que la familia sostiene acorde a sus creencias religiosas.

b) Principios y conductas específicas que practican sus amigos (vecindario).

c) La carga de valores y antivalores a los que se encuentran expuestos por largas horas a través de los medios de comunicación, en especial la televisión, la cuál les "vende" sus propios "valores" (Loera, 1999 en Martínez y Hernández, 2005).

A pesar de que el tema de los valores ha cobrado importancia en la actualidad como parte de la filosofía, los valores han estado presentes desde el inicio de la humanidad. Para el ser humano siempre han existido cosas valiosas: el bien, la verdad, la belleza, la felicidad, la virtud y a lo largo de nuestra historia han existido situaciones que se valoran más que otras o de manera diferente. Por lo anterior, el criterio para estimar ha variado a través de los tiempos: se puede apreciar de acuerdo con criterios estéticos, esquemas sociales, costumbres, principios éticos o, en otros términos, por el costo, la utilidad, el bienestar, el placer o el prestigio.

En esta interpretación de la forma de estimar lo que nos rodea se manifiesta otro problema: una confusión entre lo que son los valores y el proceso de valoración de las cosas. 


\section{Parámetros bajo los cuales se comprenden los valores en esta investigación}

El concepto de valor en el que se busca educar a los niños abarca contenidos y significados diferentes y ha sido abordado desde diversas perspectivas y teorías.

La visión subjetivista considera que los valores no son reales, no valen en sí mismos, sino que son las personas quienes les otorgan un determinado estima, dependiendo del agrado o desagrado que producen. Desde esta perspectiva, los valores son subjetivos, dependen de la impresión personal del ser humano. La escuela neokantiana afirma que el valor es, ante todo, una idea. Se diferencia lo que es valioso, de lo que no lo es, dependiendo de las ideas o conceptos generales que comparten las personas. Algunos autores indican que no son concretos, no se encuentran en el mundo sensible y objetivo, sino en el pensamiento de quien los contiene; desde esta perspectiva es en la mente donde los valores se aprehenden, cobran forma y significado. En este relativismo, cada quien crea su propia jerarquía y defiende sus valores, en este sentido, es posible afirmar que los valores de los hijos ya no concuerdan con los valores de los padres; perspectiva que no se comparte en esta investigación.

La escuela fenomenológica, desde una perspectiva idealista, considera que los valores son ideales y objetivos; valen independientemente de las cosas y de las estimaciones de las personas. Así, aunque todos sean injustos, la justicia sigue teniendo valor.

En sentido humanista, se entiende por valor lo que hace que un hombre sea tal, sin lo cual perdería la humanidad o parte de ella. El valor se refiere a una excelencia o a una perfección. Por ejemplo, se considera un valor decir la verdad y ser honesto; ser sincero en vez de ser falso; es más valioso trabajar que robar. La práctica del valor desarrolla la humanidad de la persona, mientras que el contravalor lo despoja de esa cualidad (Vásquez, 1999, p. 3). Desde un punto de vista socio-educativo, los valores son considerados referentes, pautas o abstracciones que orientan el comportamiento humano hacia la transformación social y la realización de la persona. Son guías que dan determinada orientación a la conducta y a la vida de cada individuo y de cada grupo social. Y es bajo esta visión del valor, en la cual este trabajo se desarrolla.

La ciencia que estudia a los valores es la Axiología, rama de la metafísica proviene del griego axiós = valioso, estimable, digno de ser honrado y del logos que significa palabra, estudio o ciencia (Ferrater, 1981, p. 264), así la axiología es la ciencia que estudia los valores o teorías de los valores, que profundiza por un lado la esencia del valor, la clasificación de los valores y el proceso de valoración o juicios de valor (López, 2000, p. 45).

Desde el punto de vista etimológico valor proviene de valere (Gómez, 2001, p. 708) ser fuerte, ser potente. Esta potencia es lo que hace que cada ser se realice como tal (ser).

López (2000, p. 23) afirma que un valor es una perfección real o posible, real porque está enraizada en el ser de todo lo que existe y posible porque está potencialmente presente y puede actualizarse. Esta perfección procede de la naturaleza que se encuentra en el ser y en la razón de ser. El ser es el soporte de los valores pero también la razón de ser, su misión, lo que le da sentido en esta realidad. Desde esta perspectiva los valores son inamovibles; en cada ser no cambia su esencia, mientras permanece siendo lo que es.

El valor emana de la esencia de las cosas, es algo sin lo cual el ser no podría ser lo que es, o no existiría como tal; el valor es como se identifica cada cosa, es algo que no puede cambiar en cada ser, es aquello que le brinda a cada ser lo que es. Por ejemplo, si se tiene diferentes tipos 
de zapatos, ellos poseen un color distinto, forma, medida, precio, textura, diferentes, si se pone fuera todas las cosas que cambian de los zapatos, qué queda, el valor de los zapatos es proteger el pie al caminar. De la misma manera, el valor del fuego es la capacidad de calentar y el valor de la sal es, ser salada, porque si no sala, simplemente no es sal.

El ser humano además de ser un ser racional es también un ser que valora. La relación que el hombre establece con el mundo se gesta a partir de la valía que éste establece. En este sentido hay dos actos importantes al valorar: primero que se capte la perfección, y después el que merece ser estimada (López, 2000).

Valoración es el acto de valorar o sea, evaluar, estimar, apreciar. Valorar es descubrir el valor de algo. Es captar y expresar de manera propia la perfección de la realidad. Es captar lo objetivo de modo personal siempre que incluya el respeto a la realidad. Es un planteamiento subjetivo pero no subjetivista.

Las valoraciones son producto de cambios y transformaciones a lo largo de la historia, es el nivel de estimación que se le asigna a un objeto; la valoración surge con un especial significado en un momento, para después cambiar o desaparecer en una época distinta. De esto resulta la dificultad no sólo de precisión en los términos de lo que los valores son, sino de tener claro cuál es el proceso de valoración en los seres humanos en la actualidad y cómo se desarrolla éste en el binómico familiaescuela.

Otra de las dificultades que se percibe es la incoherencia de quienes educan en valores a los niños, ya que sus modelos a seguir -a saber padres y maestros principalmente- con frecuencia carecen de consistencia. Esta incongruencia que perciben los niños día a día en la actuación de dichos educadores no sólo los confunde y les resta autoridad, sino que pierde toda la fuerza que se requiere para vivir auténticamente una vida recta.

\section{Metodología de investigación para este estudio a partir de la investigación acción}

El trabajo en el colegio se inició con una evaluación exploratoria para determinar los alcances del modelo educativo en valores de la escuela, para lo cual se llevaron a cabo entrevistas semiestructuradas que arrojaron los siguientes resultados: la escuela está trabajando tres propuestas de valores que se han puesto en marcha en diferentes momentos y en distintos espacios, pero sin ninguna integración ni conocimiento profundo de ellas, lo que causa confusiones dentro del ambiente escolar.

Con base en la exploración inicial se identificaron las siguientes necesidades:

a. Iniciar el diseño de un proyecto educativo que integre los tres programas en valores: Secretaría de Educación Pública, Montessori y Sathya Sai -formación valoral- para que exista una integración de los valores que se proponen en cada programa.

b. Incorporar a todo el personal de la escuela que maneja el programa de valores en el diseño para que se apropien de sus principios teóricos y los articulen con los fines que la escuela persigue.

c. Integrar a los padres de familia en este proceso, ya que ello permitiría trabajar en casa y en la escuela bajo los mismos conceptos de valores, lo cual proporcionaría a los niños una guía coherente y continua en la escuela y la casa. Los hallazgos de este estudio preliminar permitieron el siguiente objetivo de investigación:

Objetivo general: Diseñar una propuesta en valores que sea equivalente en la familia y en la escuela donde participen los padres, maestros, alumnos y autoridades para que los niños cuenten con un modelo congruente de vivencia en valores en los espacios educativos en los que se desarrollan. 
A continuación se muestra la metodología empleada en este proyecto y su justificación. La metodología propuesta en esta investigación para la construcción de la propuesta integradora de formación en valores del colegio es la investigación acción por razones muy valiosas que muestra el trabajo de Bonifacio (2005) en el que se cuestiona si la educación y los valores son en realidad dos cosas distintas o una supone a la otra "un nuevo sentido formativo de la escuela y de su eficacia social y pedagógica se ha generalizado en el discurso educativo para dejar claro que la educación es, por naturaleza, una cuestión de valores, un proceso de formación moral (Bonifacio, 2005, p. 9), este principio se ha de trabajar en cada profesor para hacer conciencia si los valores que subyacen en su práctica docente coinciden con el programa educativo de formación en valores a construir por y para la comunidad escolar.

La investigación acción "hace referencia a una amplia gama de estrategias realizadas para mejorar el sistema educativo y social... es una familia de actividades que se realiza principalmente por profesores en sus propias aulas con fines como: su autodesarrollo profesional, la mejora de los programas educativos ...Estas actividades tienen en común el desarrollo de las estrategias de acción que son implementadas, sometidas a observación, reflexión y cambio" (Latorre, 2005, p. 23). Elliott la define como "el estudio de una situación social con el fin de mejorar la calidad de la acción dentro de la misma" (Elliott, 1993, en Latorre, 2005, p. 24) y Kemmis la concibe "no sólo como una ciencia practica y moral, sino también como ciencia crítica definiéndola como una forma de indagación auto reflexiva por quienes participan: profesorado, alumnos o dirección en una situación social (incluyendo las educativas) para mejorar la racionalidad de: las propias prácticas educativas y la comprensión sobre las mismas..." (Kemmis, 1988, en Latorre, 2005, p. 25).
"El objetivo fundamental de la investigación acción es mejorar la práctica..." (Elliot, 2000, p. 67) y señala en este mismo texto el autor, cómo la investigación acción es sugerida para el estudio de los valores, afirmando que la reflexión dirigida a la puesta en práctica de los valores puede describirse como filosofía práctica, se ha de llevar la atención hacia el papel que las críticas reflexivas de las interpretaciones de valor incluidas en la práctica pueden desempeñar para mejorarla (cfr. Elliot, 2000, p. 69).

La metodología de la investigación acción participativa permite al investigador analizar, junto con los participantes y mediante ciertas técnicas grupales, los cuestionamientos fundamentales del tema -en este caso la formación de valores- y las posibles alternativas para resolverlos, a través de la participación plena de los interesados. En su metodología se va desarrollando de manera recurrente por momentos o etapas que se van repitiendo en espiral: planeación, acción y reflexión participativa. El análisis de los conflictos que surgen es vital. La recuperación de la información se recoge en bitácoras, donde se establece cómo va evolucionando, tanto la tarea como los procesos grupales asociados a su ejecución (Eisenberg, 2006, en Hirsch, 2006, p. 458). Esta es una explicación de lo que se llevó a cabo en esta investigación. Este estudio parte de quienes conocen su comunidad y viven diariamente la problemática; brinda conocimiento desde su reflexión permitiendo con ello, que su participación activa en las soluciones resulte natural llevarla a la práctica y no una imposición ajena que hay que aplicar en su realidad educativa.

Elliott (2000) narra algunos casos de la aplicación de esta metodología como "Ford Teaching Projet" patrocinado por fundación Ford cuyo equipo de investigación se encontraba conformado por él y Clem Adelman, participaron en el proyecto 40 profesores de 12 escuelas, desarrollaron una investigación-acción sobre problemas conductuales en sus clases. Otro 
caso narrado en el mismo texto, es el del Humanites Curriculo Project donde se realizó una reforma al currículo oficial en materia de Humanidades llevado a cabo en el School Council y dirigido por Elliot y Lawrence; además de otros casos presentados. En las aportaciones de ambos sucesos se relatan:

se les pedía que los profesores desarrollar estrategias de acción a partir de los datos observados en su clase,... realizábamos un análisis comparativo de las transcripciones de las grabaciones con el objetivo de generar hipótesis que pudieran generalizarse a otras aulas o escuelas... o ayudamos a que los maestros mejoraran su práctica educativa (Elliot, 2000, pp. 35-37).

Cuando se encuentran estos resultados positivos alientan a seguir el ejemplo y crear un grupo laborioso que permita el alcance de los propios objetivos, nada ajenos a los presentados en estos casos. Se encontraron en las conclusiones del texto anteriormente señalado, algunas ventajas de esta metodología de investigación acción

"puede considerarse como un conjunto de estrategias para facilitar juicios, decisiones y acciones reflexivas a cargo de todas las partes, cuyas actividades configuren e influyan en los procesos de enseñanza aprendizaje. Al fomentar la práctica reflexiva entre los profesores del aula, los directivos y los consumidores de la enseñanza: alumnos y padres, fomentamos el aprendizaje mediante la investigación-acción de todas las partes implicadas" (Elliot, 2000, pp. 58-59).

esta es una de las razones por la que se eligió este método de investigación para crear un equipo con padres, maestros, directivos, alumnos e investigador y juntos desde la reflexión e instrumentos aplicados a la práctica diaria en el colegio se construya un programa que responda a las necesidades y los objetivos planteados para la formación valoral en la comunidad educativa del Colegio Montessori de Tepuente.

Otra de las posibilidades de esta metodología es la posibilidad de autoevaluación de los profesores, hacer conciencia de cómo se desarrolla en realidad su práctica y que ellos mismos propongan medios de mejora operativa, además es necesario que establezcan "la relación entre el comportamiento normativo del docente y la conducción del proceso de enseñanza" (Fierro y Carbajal, 2005, p. 32) porque en todo proceso de enseñanza -en forma conciente o no-el profesor imprime sus propios valores y forma de concebir lo que significa educar.

Otro trabajo revisado fue el de PIPES (Fundación de ayuda contra la drogadicción, s. f.) que es un plan de intervención educativo escolar que pone en marcha estrategias para la prevención del consumo de drogas a través del compromiso comunitario escolar. De él se rescatan tres elementos para la propuesta que esta investigación se plantea: 1. Partir del carácter integral de la personalidad del individuo, 2. Abierto a la participación e innovación del profesorado en su aula y en su propio centro escolar y 3. Adaptarlo al sistema educativo vigente.

En conclusión, serán los autores Elliott (2000), Latorre (2005) y Barabtarlo y Zedansky, A. (1995) de quienes ya se explicó sus posiciones metodológicas de la investigación acción, quienes fundamentarán la parte metodológica de ésta propuesta de investigación

Categorías del estudio: De acuerdo al acercamiento exploratorio se proponen las siguientes categorías:

a) Nivel de congruencia entre valores propuestos en los 3 programas del colegio y su manejo en la familia.

b) Integración de la construcción de la propuesta de formación en valores por los directivos y docentes del colegio a partir de la investigación acción.

Supuesto: En las actividades educativas de los guías del colegio Montessori de Tepuente la actividad de la investigación en el aula se encuentra desvinculada de la docencia. 
Diseño de la investigación: El enfoque del estudio es cualitativo, con la modalidad de investigación acción, cuyo alcance será descriptivo.

Sujetos: Alumnos de 6 a 12 años de dos grupos del Colegio Montessori, Maestros del colegio; Directora del colegio y Padres de familia de los alumnos.

Muestra: Se utilizará una muestra no probabilística que considera a la población voluntaria tanto en alumnos, guías y padres de familia no menor al $20 \%$ del universo.

Procedimiento: Se realizará en tres fases: Fase 1. Evaluación diagnóstica del programa educativo de formación en valores del Colegio Montessori a través de la investigación en acción con la participación de docentes, directivos, alumnos y padres de familia. Fase 2: Trabajo de campo con aplicación y análisis de tres instrumentos: a. Entrevistas semiestructuradas con los alumnos de cada grupo elegidos al azar, para analizar las vivencias en el programa de valores; b. Registros de observación para recuperar evidencias sobre qué tanto conocen el fundamento y funcionamiento del programa de valores; c. Elaborar escalas de actitud para detectar el nivel de coherencia sobre los valores entre los padres de familia, maestros y alumnos. Fase 3. Análisis de resultados y diseño de propuesta inicial.

\section{Resultados}

Los docentes del Colegio Montessori de Tepuente en un 29\% realizan una adaptación de los contenidos de los programas propuestos por la SEP y los van adaptando de acuerdo al avance de sus grupos de forma muy inmediata a su aplicación (entre 1 semana, el día previo hasta el momento mismo de la implementación).

Por parte de la autoridad educativa hay solicitud de la programación docente pero no existe revisión alguna de la misma en el único caso de elaboración de la misma.
Los guías expresaron motivación en la aplicación de instrumentos para el desarrollo de una investigación de su propia práctica pero en el momento de ejercitarla manifestaron una limitante al no tener habilidades para implementarla y una carga excesiva de trabajo que les generaba una tensión más por el registro de los hechos que se solicitaban en los instrumentos. En general han concebido que su responsabilidad recaiga en la atención de los alumnos y la implementación del programa escolar.

Otra limitación hacia el desenvolvimiento de su tarea fue que conocen los conceptos y prácticas educativas pero en muy pocos casos lo aplican para el buen desarrollo de su labor, ellos se conforman por hacer lo mínimo necesario para que se lleve a cabo la enseñanza y solo en dos casos reportaron iniciativas de registros personalizado más práctico para el avance de sus alumnos.

Existen limitaciones en su formación docente en un $29 \%$ que no cuentan con la preparación específica como guías Montessori, en un 95\% no planean los contenidos educativos, pero al percibir ellos esta necesidad sí se encuentran con disposición de recibir cursos de formación y actualización a lo que el colegio ha implementado algunos cursos y talleres para cubrirlo.

Los profesores no se interesan por la investigación acción. Existieron limitaciones metodológicas en los guías que les llevaron a tener que realizar un esfuerzo continuo con base en correcciones constantes que se fueron realizando a sus instrumentos hasta llegar a comprender su uso y llevarlo a cabo correctamente.

Los docentes afirman muy a menudo que los manuales sobre educación son muy teóricos y de poca utilidad en la práctica porque no responden a sus necesidades ya que por lo común, los investigadores que los elaboran no ejercen la actividad docente, sin embargo, dichos investigadores afirman que es la falta de conocimientos e ineptitudes de los profesores para aplicarlas lo que limita los resultados, al respecto Reyna 
afirma "existe un divorcio entre la teoría y la práctica educativa; divorcio que se traduce en individuos dedicados exclusivamente a producir teoría y otros encargados de consumirla" (Reyna, 2007, p. 9).

Existen pocas habilidades y motivación por parte del docente para vincular la investigación acción participativa a su propia practica en el aula.

\section{Conclusiones y aportación}

La apatía mostrada hacia la investigación acción de los docentes se debe a que la investigación no es una práctica común en ellos, por lo que la reconocen como ajena a su labor y como no se encuentra en ello una remuneración monetaria, existe escaso interés de su ejercicio en el aula.

La investigación acción es un procedimiento que permite interrelacionar la teoría y práctica educativa; los problemas surgen de la misma práctica educativa y son los docentes mediante un estudio de investigación, quienes proponen las estrategias para abordar dicha problemática e irla resolviendo a través de su labor en el aula. Es así como la docencia y la investigación confluyen en la misma persona dedicada a la educación.

La investigación acción funciona como una herramienta eficaz para mejorar la calidad educativa en la educación básica siempre y cuando el docente se encuentre motivado y en disposición de desarrollarla en su tarea educativa diaria.

Los propuesta de intervención en el aula a través de la investigación acción es un proyecto que suscita el desarrollo de la docencia profesionalizada a través del manejo adecuado de los problemas que se van generando en las clases, las relaciones, la enseñanza aprendizaje, en el proceso de planeación hasta la evaluación, permitiendo que el profesor sea capaz de auto-observarse constantemente y sea crítico ante su propio actuar.

La formación docente y su motivación en el trabajo influyeron en tener una actitud conflictiva de aceptación al desarrollo de la investigación en su acción educativa.

En el proceso de enseñanza, el docente es el protagonista primordial ya que de su actuar diario depende en gran medida la calidad con la que se educa a los estudiantes, en el caso de los guías del colegio Montessori de Tepuente perciben su labor como un trabajo seguro; la permanencia de la planta actual de profesores, evidencian el compromiso con la institución, sin embargo, todos desean mejores condiciones de trabajo y mejores salarios similares a los de otros colegios Montessori; en cuanto a la formación específica como educadores el $88 \%$ de ellos manifiesta que su proyecto está encaminado a la labor que desempeñan; pero en otros casos falta completar sus estudios, la poca preparación para el Método Montessori -en un 28\% solamente- es muestra clara de la necesidad de seguirse formando en este rubro, el tiempo con el que cuentan para desarrollar su actividad en un $82 \%$ de ellos es amplio y el contexto socioeconómico al que pertenecen es medio o medio bajo; en uno de ellos le exige buscar otra actividad para su complemento económico.

El supuesto del que partió esta investigación se confirma al encontrar desvinculada la labor docente de la práctica investigativa y muy poco interés de los docentes por realizar el esfuerzo por desarrollarla.

No existieron medios de formación en la investigación que permitiera que en el ejercicio de la labor se les desarrollaran estas habilidades con facilidad. Sin embargo, a través de este estudio, los profesores han desarrollado la habilidad de la autoobservación a partir de elementos que se requería conocer y profundizar, que los llevaron a hacerse concientes de cómo realizaban realmente su práctica y desarrollar evidencias para hacer una valoración mucho más objetiva de la misma y estrategia de mejora en su caso.

Ante los grandes problemas y necesidades de mejora educativa que presenta hoy la educación mexicana, la investigación 
acción se presenta como una posibilidad viable y accesible, es necesario que se implemente en las instituciones esta metodología de forma continua para que cada vez mas profesores se vean beneficiados y esto redunde en mayor calidad educativa en la educación de nuestros días.

\section{Referencias bibliográficas}

Aula Santillana. (1996). Diccionario de las ciencias de la educación. Madrid, España: Editorial Santillana.

Barabtarlo y Zedansky, A. (1995). Investigación acción. Una didáctica para la formación de profesores. México: UNAM Centro de Investigaciones y servicios educativos.

Bartau, I., Maganto, J. y Etxeberría, J. (s.f.). Los programas de formación de padres: una experiencia educativa. Revista Iberoamericana de Educación. Extraído el 15 agosto de 2006 de http://www.rieoei.org/par com2.htm

Bonifacio, B. (2005, enero-marzo). Educación y valores: una búsqueda para reconstruir la convivencia. Revista Mexicana de investigación Educativa, 024(10), 9-14.

Centro de cooperación regional para la educación de adultos en América Latina y el Caribe. [CREFAL]. (2000). La escuela y la Familia. Libros de papá y mamá. Michoacán, México: Comisión Nacional de los libros de texto gratuitos (CONALITEG).

Díaz, A. (2006). La Educación en valores: Avatares del currículo formal, oculto y los temas transversales. Revista Electrónica de Investigación Educativa, 1(8), 1-15. Extraído el 2 septiembre, 2006 de http://redie.uabc.mx/vol8no1/ contenido-diazbarriga2.html

Elexpuru, I. y Medrano, C. (2001). Desarrollo de los valores en las instituciones educativas. Bilbao: Universidad de Deusto.

Elliot, J. (2000). El cambio educativo desde la investigación-acción. ( $3^{\mathrm{a}}$ ed.). Madrid, España: Morata.

Ferrater, M. (1981). Diccionario de Filosofía. ( $3^{\mathrm{a}}$ ed.). Barcelona, España: Alianza diccionarios.

Fierro, C. y Carbajal, P. (2005). Mirar la práctica docente desde los valores. $\left(2^{\circ}\right.$ ed.). México: Gedisa.

Fundación de ayuda contra la drogadicción [PIPES ]. (s. f.). Plan integral de prevención escolar del consumo de drogas. México: Edición particular.

Gómez, G. (2001). Breve Diccionario etimológico de la lengua española. México: Fondo de Cultura Económica.

Hirsch, A. [Coord.]. (2006,) Educación y valores, Tomo I, II y III. México: Ediciones Gernika.

Latapí, P. (2004). El debate sobre los valores en la escuela mexicana. México: Fondo de Cultura Económica.

Latorre, A. (2005). La investigación acción. Conocer y cambiar la práctica educativa. Barcelona, España: Editorial Graó.

López, A. (2000). Educación en valores, educación en virtudes. México: CECSA.

Martínez, P. y Galíndez, N. E. (2003). Familia y entorno. Implicación de la familia en el entorno escolar. Bilbao, España: Fundación Horreum Fundazioa. 
Martínez, T. y Hernández, J. (2005). Elementos filosóficos y teóricos para la investigación educativa en valores. Una primera aproximación. En A. Hirsch Adler (Coord.), Formación valoral y ciudadana, Tomo III ( $p p$. 41-65). México: Gernica.

Martínez, T. y Hernández, J. (2005). Hacia la identificación de los elementos teórico-metodológicos para la investigación educativa en valores. En A. Hirsch Adler (Coord.), Formación valoral y ciudadana Tomo II. México: Gernica.

Organización para la Cooperación y el Desarrollo Económicos [OCDE]. (2002). Conocimientos y Aptitudes para la vida. Resultados de PISA 2000. México: Aula XXI Santillana.

Ortega, P. y Mínguez, R. (2001). La educación Moral del ciudadano del hoy. Barcelona, España: Paidós.

Reyna, F. (2007). Investigación acción en el aula. México: Universidad La Salle Pachuca.
Rocher, Guy. (1983). Introducción a la sociología general. Barcelona: Herder.

Sañudo, L. (1997, Abril-Junio). Los programas de intervención una modalidad para investigar en la educación. Educar, 3. Extraído el 13 julio, 2006 de http://educacion.jalisco.gob.mx/ consulta/educar/01/01Lya.html

Schmelkes, S., Linares, M. E. y Delgado, M. A. (1995). Educación, Cultura y Procesos Sociales. México: Consejo Mexicano de Investigación Educativa A.C.

UNESCO. (2006). Informe del avance del plan piloto del programa educación en valores humanos 2006-20007, Maracay: Universidad Pedagógica Experimental Libertador.

Vásquez, E. (1999). Reflexiones sobre el valor. Suplemento Cultural de Últimas Noticias, 1 (Suppl. 606), 1-3.

Yurén, T., Navia, C. y Saenger. [Coords]. (2005). Ethos y autoformación del docente. Análisis de dispositivos de formación de profesores. BarcelonaMéxico: Ediciones Pomares. 
